\title{
The Tensor Product of Polynomials
}

\author{
Ruth Schwingel
}

\section{CONTENTS}

1. Introduction

2. The Tensor Product

3. Cases $\mathrm{I}_{22}$ and $\mathrm{I}_{32}$

References
Using Gröbner basis algorithms in MAGMA we find necessary and sufficient conditions for a polynomial of degree 6 over any field to be the tensor product of two polynomials, one of degree 3 and one of degree 2.

\section{INTRODUCTION}

In order to determine whether there exists a tensor decomposition of the natural module for a matrix group $G$ over a field $K$ it proved to be useful to decide whether or not there exists a tensor decomposition of the characteristic polynomial of $g \in G$ [Leedham-Green and O'Brien 1997]. This latter problem was the motivation for the present work.

Let $h$ be a univariate polynomial of degree $d$ over an algebraically closed field $K$. If $d=m+n$ then clearly $h$ is the product of two polynomials over $K$ of degrees $m$ and $n$. But if $d=m n$, with $m, n>1$, then $h$ is the tensor product (as defined below) of two polynomials, one of degree $m$ and the other of degree $n$, if and only if the coefficients $c_{1}, \ldots, c_{d}$ of $h$ define an element $\left(c_{1}, \ldots, c_{d}\right)$ in some $(m+n-1)$ dimensional variety $V \subset K^{d}$. This variety is determined by a prime ideal $I_{m n}$ in the ring $K\left[c_{1}, \ldots, c_{d}\right]$. The ideal $I_{22}$ is easily computed by hand and the ideal $I_{32}$ is just within the range of machine computation.

\section{THE TENSOR PRODUCT}

Given two monic polynomials

$$
f(x)=x^{m}-a_{1} x^{m-1}+\cdots+(-1)^{m} a_{m}
$$

with zeros $\alpha_{1}, \ldots, \alpha_{m}$ and

$$
g(x)=x^{n}-b_{1} x^{n-1}+\cdots+(-1)^{n} b_{n}
$$

with zeros $\beta_{1}, \ldots, \beta_{n}$ in $K[x]$, the tensor product of $f(x)$ and $g(x)$ is the monic polynomial $h(x)$ of degree 
$m n$ with roots $\alpha_{j} \beta_{k}$ for $1 \leq j \leq m, 1 \leq k \leq n$; that is,

$$
h(x)=x^{m n}-c_{1} x^{m n-1}+\cdots+(-1)^{m n} c_{m n},
$$

with $c_{i}$ the $i$-th elementary symmetric function in $\alpha_{j} \beta_{k}$, for $1 \leq j \leq m$ and $1 \leq k \leq n$.

Let

$$
\begin{aligned}
p_{i}(f) & =\sum_{j=1}^{m} \alpha_{j}^{i}, \\
p_{i}(g) & =\sum_{k=1}^{n} \beta_{k}^{i}, \\
p_{i}(f \otimes g) & =\sum_{j, k}\left(\alpha_{j} \beta_{k}\right)^{i} \\
& =\left(\sum_{j=1}^{m} \alpha_{j}^{i}\right)\left(\sum_{k=1}^{n} \beta_{k}^{i}\right)=p_{i}(f) p_{i}(g)
\end{aligned}
$$

be the $i$-th power sums of $\alpha_{j}, \beta_{k}$ and $\alpha_{j} \beta_{k}$, where $1 \leq j \leq m$ and $1 \leq k \leq n$, respectively.

We can compute the $i$-th power sum $p_{i}$ in terms of $\left\{e_{1}, \ldots, e_{i}\right\}$ by using Newton's Formula [Macdonald 1995, p. 23],

$$
n e_{n}=\sum_{r=1}^{n}(-1)^{r-1} p_{r} e_{n-r},
$$

where $e_{j}$ is the $j$-th elementary symmetric function. Then by a simple algorithm we can compute the $c_{i}$ 's in terms of $\left\{a_{j}: 1 \leq j \leq m\right\}$ and $\left\{b_{k}: 1 \leq k \leq n\right\}$.

The weight in the $x$ 's of a monomial $x_{1}^{\varepsilon_{1}} \cdots x_{m}^{\varepsilon_{m}}$ is defined by $w=\sum_{i=1}^{m} i \cdot \varepsilon_{i}$. Each $c_{i}$ is then a homogeneous polynomial of weight $i$ in both the $a_{j}$ 's and the $b_{k}$ 's.

In general, the condition that the polynomial $h$ should have a tensor factorisation with factors of degrees $m$ and $n$ is the condition that the coefficients of $h$ define an element $\left(c_{1}, \ldots, c_{m n}\right)$ in the variety $V \subset K^{m n}$ determined by an homogeneous ideal $I_{m n} \subset K\left[c_{1}, \ldots, c_{m n}\right] . I_{m n}$ is the kernel of the homomorphism from $K\left[c_{1}, \ldots, c_{m n}\right]$ into

$$
K\left[a_{1}, \ldots, a_{m}, b_{1}, \ldots, b_{n}\right]
$$

taking each $c_{i}$ to the corresponding polynomial in the $a_{j}$ 's and $b_{k}$ 's. Being the kernel of an homomorphism into a domain, $I_{m n}$ is a prime ideal, hence the variety $V$ is irreducible.
To determine the dimension of $V$ we consider the factorisation

$$
h(x)=f(x) \otimes g(x)=\prod_{j, k}\left(x-\alpha_{j} \beta_{k}\right)
$$

giving the polynomial functions $\varphi_{j k}: K^{m+n} \rightarrow K$ defined by

$$
\varphi_{j k}\left(\alpha_{1}, \ldots, \alpha_{m}, \beta_{1}, \ldots, \beta_{n}\right)=\alpha_{j} \beta_{k} .
$$

It is easy to see that the $m+n-1$ elements $\varphi_{11}$, $\ldots, \varphi_{m 1}, \varphi_{12}, \ldots, \varphi_{1 n}$ form a maximal set of algebraically independent elements over $K$, hence the dimension of $V$ is $m+n-1$. For more details on the theory of varieties see [Cox et al. 1997, Chapters 4, $5,9]$.

\section{CASES $I_{22}$ AND $I_{32}$}

It is easy to prove that $I_{22}$ is a principal ideal with generator of weight 6 . The coefficients are

$$
\begin{aligned}
& c_{1}=a_{1} b_{1}, \\
& c_{2}=a_{2} b_{1}^{2}+a_{1}^{2} b_{2}-2 a_{2} b_{2}, \\
& c_{3}=a_{1} a_{2} b_{1} b_{2}, \\
& c_{4}=a_{2}^{2} b_{2}^{2},
\end{aligned}
$$

so that the generator $c_{1}^{2} c_{4}-c_{3}^{2}$ can be easily obtained.

The problem of finding a set of generators for $I_{32}$ proved surprisingly harder. This is a classical Gröbner basis problem. Considering the polynomial parametrization

$$
\begin{aligned}
& c_{1}=q_{1}\left(a_{1}, \ldots, a_{m}, b_{1}, \ldots, b_{n}\right), \\
& c_{d}=q_{d}\left(a_{1}, \ldots, a_{m}, b_{1}, \ldots, b_{n}\right),
\end{aligned}
$$

let $I$ be the ideal

$$
\begin{aligned}
I=\left\langle c_{1}-q_{1}, \ldots, c_{d}-q_{d}\right\rangle \\
\\
\subset K\left[a_{1}, \ldots, a_{m}, b_{1}, \ldots, b_{n}, c_{1}, \ldots, c_{d}\right] .
\end{aligned}
$$

Then the ideal $I_{m n}$ is the $(m+n)$-th elimination ideal $I_{m n}=I \cap K\left[c_{1}, \ldots, c_{d}\right]$, and the Elimination Theorem [Cox et al. 1997, $\S 5.3$, Theorem 1] proves that if $B$ is a Gröbner basis for $I$ with respect to lex order where $a_{1}>\cdots>a_{m}>b_{1}>\cdots>b_{n}>c_{1}>$ $\cdots>c_{d}$ then the set $B_{m n}=B \cap K\left[c_{1}, \ldots, c_{d}\right]$ is a Gröbner basis for $I_{m n}$.

We were unable to get the calculation to complete on any Gröbner basis package. Clearly $I_{m n}$ is defined over $\mathbb{Q}$ (equivalently over $\mathbb{Z}$ ). Working over 
GF(2) without using Gröbner techniques it was possible, using MAGMA [Bosma and Cannon 1993], to find homogeneous elements of $I_{32}$ that we believed to form a generating set. The conjecture was later confirmed when Allan Steel showed us how to carry out the complete calculation using the Gröbner basis in MAGMA, working over $\mathbb{Q}$. This was done by defining the polynomial ring

$$
P=\mathbb{Q}\left[a_{1}, a_{2}, a_{3}, b_{1}, b_{2}, c_{1}, \ldots, c_{6}\right]
$$

with elimination order [Cox et al. 1997, p. 72], then defining the ideal $I=\left\langle c_{1}-q_{1}, \ldots, c_{6}-q_{6}\right\rangle$ in $P$ and determining its Gröbner basis $B$. A Gröbner basis $D$ for the elimination ideal $I_{32}$ is obtained by taking the images of the basis elements $b \in B$ under the homomorphism $\psi: P \rightarrow K\left[c_{1}, \ldots, c_{6}\right]$ defined by $\psi\left(a_{j}\right)=\psi\left(b_{k}\right)=0$, and $\psi\left(c_{i}\right)=c_{i}$. Eliminating redundancies in $D$ a minimal generating set for $I_{32}$ is obtained. The conclusion is that a minimal generating set for $I_{32}$ contains 16 homogeneous polynomials of weights 19 to 30, each being the sum of at least 28 monomials.

It is hoped that new development of MAGMA Gröbner basis code will enable us to compute a free homogeneous resolution of the subring $M$ of

$$
K\left[a_{1}, a_{2}, a_{3}, b_{1}, b_{2}\right]
$$

generated by the images of $c_{1}, \ldots, c_{6}$. Preliminary calculations suggest a resolution of length five

$$
0 \rightarrow F_{5} \rightarrow F_{4} \rightarrow F_{3} \rightarrow F_{2} \rightarrow F_{1} \rightarrow F_{0} \rightarrow M \rightarrow 0,
$$

where the $F_{i}$ are free modules over $K\left[c_{1}, \ldots, c_{6}\right]$ as follows: $F_{0}$ of rank 1 with a generator of weight 0 ,
$F_{1}=I_{32}, F_{2}$ generated by 34 polynomials of weights 24 to $35, F_{3}$ by 29 polynomials of weights 28 to 38 , $F_{4}$ by 12 polynomials of weights 33 to 40 and $F_{5}$ by two polynomials of weights 39 and 41 .

The CPU time required for the calculation of the generators for $I_{32}$ using MAGMA Version 2.3-1 on a Pentium II PC was 21 minutes. The polynomials are available from ftp://ftp.maths.qmw.ac.uk/pub/ crlg/poly33.

We have been unable to produce any reasonable bound to the number of generators of $I_{m n}$, or to obtain any information about the weights of the elements of a minimal generating set, except for $I_{22}$ and $I_{32}$, and have no theoretical explanation for the results obtained in these two particular cases. In particular it would be interesting to have some insight into the cohomological dimension of $M$.

\section{REFERENCES}

[Bosma and Cannon 1993] W. Bosma and J. J. Cannon, Handbook of MAGMA functions, School of Mathematics and Statistics, University of Sydney, Sydney, 1993.

[Cox et al. 1997] D. Cox, J. Little, and D. O'Shea, Ideals, varieties, and algorithms, 2nd ed., Springer, New York, 1997.

[Leedham-Green and O'Brien 1997] C. R. LeedhamGreen and E. A. O'Brien, "Recognising tensor products of matrix groups", Internat. J. Algebra Comput. 7:5 (1997), 541-559.

[Macdonald 1995] I. G. Macdonald, Symmetric functions and Hall polynomials, 2nd ed., Oxford Mathematical Monographs, Oxford Univ. Press, 1995.

Ruth Schwingel, School of Mathematical Sciences, Queen Mary and Westfield College, University of London, Mile End Road, London E14NS, UK (R.Schwingel@qmw.ac.uk) 